\title{
An Unusual Case of Antiphospholipid Syndrome Presenting as Chorea
}

\author{
Anudeep Yelam ${ }^{1}$, Pradeep C. Bollu ${ }^{1}$ \\ 1. Neurology, University of Missouri, Columbia, USA
}

Corresponding author: Anudeep Yelam, anudeepyelam@gmail.com

\begin{abstract}
Movement disorders are rarely reported in association with antiphospholipid syndrome (APS). Although it is a rare manifestation in APS, chorea is the most common movement disorder. We report a case of APS in a patient who presented with hyperkinesia, tics, choreiform movements, and other dyskinetic movements involving the mouth and tongue along with behavioral changes. These abnormal movements improved with aripiprazole.
\end{abstract}

Categories: Family/General Practice, Internal Medicine, Neurology

Keywords: antiphospholipid syndrome, chorea, movement disorders

\section{Introduction}

Chorea is defined as involuntary movements that are abrupt, unpredictable, and nonrhythmic, resulting from a continuous random flow of muscle contractions [1]. It is a rare manifestation in antiphospholipid syndrome (APS) and is approximately seen in $1 \%-2 \%$ of patients with this condition. Here, we report a patient with APS who presented with chorea. We will also briefly review the neurological manifestations of APS, the mechanism of chorea in APS, and its management.

\section{Case Presentation}

A 60-year-old, right-handed male presented to the emergency room (ER) for acute behavioral changes and abnormal movements for two weeks. He is a truck driver with a remote history of encephalitis and stroke with no baseline deficits. On examination, the patient had stereotypic, "tic-like," bilateral facial twitches more prominent on the left side, constant winking of the left eye, grimacing facial expressions, seeming to indicate pain along with some tongue thrusting (like a gecko) movements. A video shot by his wife documented these movements. In addition, the patient also exhibited pressured speech and tangential thoughts. On the Montreal Cognitive Assessment (MoCA) scale, the patient scored 20/30, scoring poorly on language, word recall, and calculation.

Received 10/15/2018 Review began 10/22/2018 Review ended 10/24/2018 Published 10/29/2018

๑) Copyright 2018

Yelam et al. This is an open access article distributed under the terms of the Creative Commons Attribution License CC-BY 3.0., which permits unrestricted use, distribution, and reproduction in any medium, provided the original author and source are credited.
Given his acute behavioral changes and abnormal movements, a computed tomography (CT) scan of the head, an electroencephalogram (EEG), a lumbar puncture with cerebrospinal fluid (CSF) analysis, complete metabolic profile (CMP), complete blood counts (CBC), urine drug screen, serum, and CSF paraneoplastic panel, iron/ferritin levels, and serum copper were ordered to rule out limbic encephalitis, seizures, and infection. All the tests were unremarkable. Magnetic resonance imagining (MRI) of the brain on axial sequence showed a lacune at the level of the midbrain on T2 fluid attenuated inversion recovery (FLAIR) suggestive of an old infarct (Figure $1 A$ ).

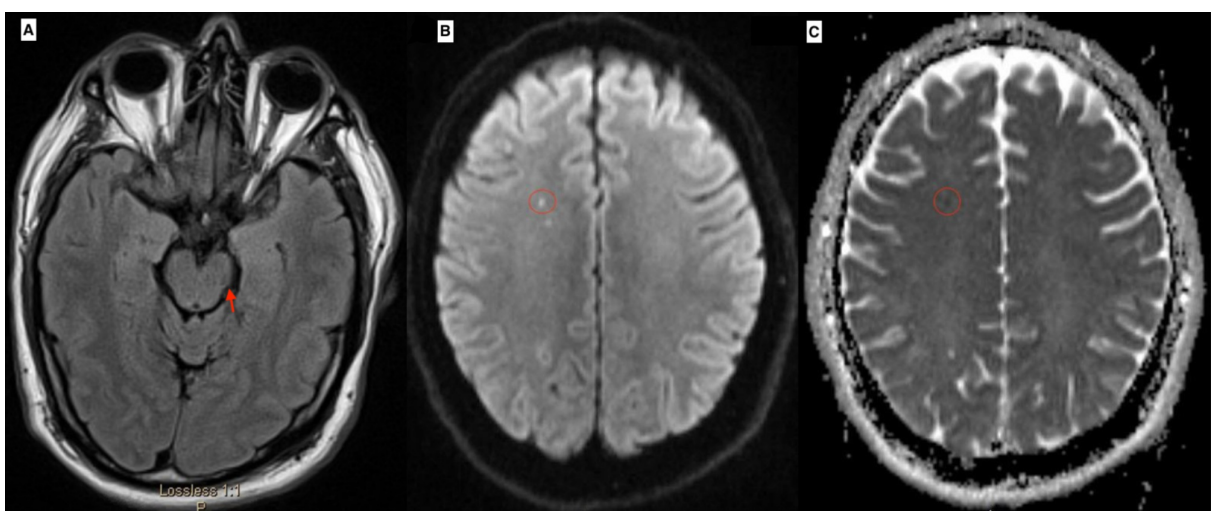

FIGURE 1: Initial magnetic resonance imaging (MRI) of the brain on axial sequence at the level of the midbrain shows a lacune (arrow) on T2 fluid 


\section{Cureus}

attenuated inversion recovery (FLAIR), suggestive of an old infarct (1A). On follow-up one month later, a tiny punctuate infarct at the level of the centrum semiovale in the right frontal region (1B) (circle) with the corresponding apparent diffusion coefficient (ADC) correlate (1C) (circle) is seen.

He was seen five days later in the outpatient neurology clinic and was noted to be much calmer and interacted well. He was started on ziprasidone by his primary care physician for suspected schizophrenia, which helped with the behavioral changes and abnormal movements. Upon further questioning, it was noted that the patient had behavioral changes many months prior to the acute onset of abnormal movements.

On his second follow-up visit one month later, it was noted that he was started on clopidogrel for deep vein thrombosis (DVT) of the right calf that happened in the interim. On examination, the patient had dyskinetic movements involving the mouth and the tongue. Choreiform and occasional high-amplitude ballistic movements are noted in the left upper and lower extremities.

Further workup, consisting of a paraneoplastic panel, vasculitis panel, myasthenia panel, CSF oligoclonal bands, comprehensive drug screen, and CT of chest, abdomen, and pelvis, revealed no abnormalities. CT angiogram of the head did not reveal irregular narrowing or dilatation. MRI of the brain revealed a tiny stroke at the level of the centrum semiovale in the right frontal region (Figures $1 B-1 C$ ). A coagulation panel showed an elevated phospholipid IgM antibody and anti-beta 2 glycoprotein 1 (B2GP1) antibody.

The diagnosis of APS presenting with chorea was made, and the patient was started on aripiprazole, which improved the symptoms.

\section{Discussion}

The diagnosis of antiphospholipid syndrome (APS) is made in this patient based on the clinical findings, history of deep vein thrombosis (DVT), imaging, elevated phospholipid IgM antibodies, and anti-beta2glycoprotein 1 (B2GP1) antibodies.

Hughes, in his original description [2], predicted the importance of neurological manifestations in patients with APS. APS can present with numerous neurological manifestations (Figure 2), among which migraine, stroke, and transient ischemic attack are the commonest [3].

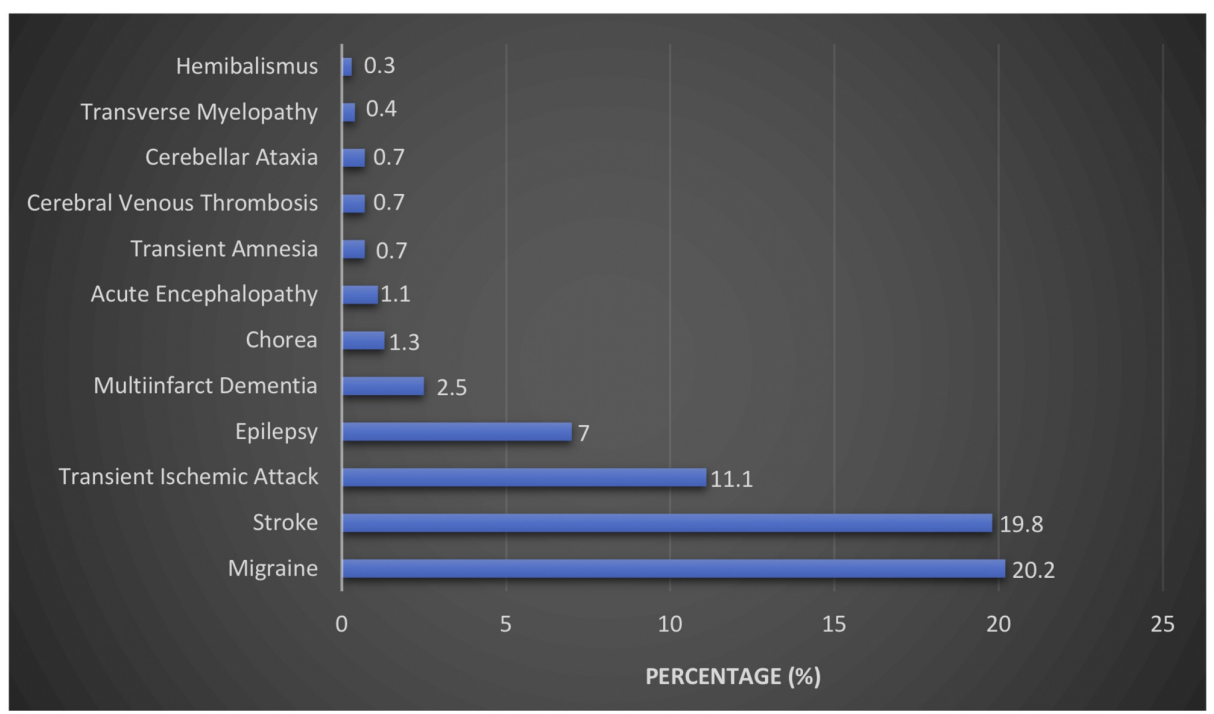

FIGURE 2: Chart demonstrating the most frequent neurologic manifestations during the evolution of antiphospholipid syndrome (APS).

Chorea is a very rare manifestation in APS and can involve any part of the body, including the head and extremities. It can be unilateral or bilateral. Cervera et al. [4] reviewed the clinical, radiological, and immunological characteristics of 50 patients with chorea and APS. Of these, $30 \%$ had primary APS and $66 \%$ 
had a single episode of chorea. The mean age of the patients in this study is $21+/-12$ years (range 6-77 years) with an onset of chorea occurring at 60 years or later in $4 \%$. CT and MRI reported cerebral infarction in $35 \%$ of these patients.

The mechanism of chorea in APS is unclear. It is thought that the antiphospholipid antibody (aPL) results in central nervous system (CNS) damage by binding to the endothelium of the brain vessels and causes endothelial dysfunction. This, in turn, increases the vascular permeability and breakdown of the blood-brain barrier (BBB), thus contributing to a hypercoagulable state and thrombus formation [5-7]. The mechanism by which aPL causes chorea is by an antigen/antibody complex binding to the phospholipids in the basal ganglia [8]. Furie et al. and his team demonstrated the presence of striatal hypermetabolism in a young woman with alternating hemichorea and primary APS [9]. During the episodes of hemichorea, the striatal glucose metabolism was noted to be elevated predominantly on the side, contralateral to the abnormal movements. Similarly, during the asymptomatic period, caudate metabolism was also significantly increased contralateral to the body side that later became symptomatic. This suggests that striatal metabolism is a functional concomitant of chorea.

There is currently no diagnostic criteria for aPL-related chorea. The presence of choreiform movements in patients with APS is suggestive of this condition. A careful clinical assessment, exclusion of other neurological conditions, combined with laboratory evaluation and neuroradiologic exams may exclude the other causes of chorea. APS diagnostic criteria [10] do not consider neurologic manifestations, although there is emerging evidence that, neurological disorders may not be uncommon in the setting of APS. The diagnosis of APS is based on a combination of clinical features and laboratory findings like anticardiolipin antibodies (aCL), anti beta2-glycoprotein 1 (B2GP1) antibodies, and Lupus anticoagulant (LA). Although the Sapporo classification criteria are designed for research purposes, they can be used as a reference for the diagnosis of APS [10].

Corticosteroids, anticoagulants, aspirin, and dopamine receptor antagonists have been reported to be effective in patients with APS chorea $[4,11]$. Neuroleptics such as haloperidol can be tried to control the hyperkinetic movements. Atypical neuroleptics can also be used as alternatives. Our patient improved on aripiprazole. In the absence of remission, the combination therapy, including neuroleptics and steroids, can be tried [4]. Anticoagulation should be reserved for thrombosis treatment and not simply for chorea in the presence of aPL, as it could result in infarction, bleeding, and death [12]. As APS in an autoimmune disorder, the use of immunomodulatory agents has been proposed [13]. But, there is a lack of enough evidence for the selection of specific immunomodulators. Hydroxychloroquine and statins have been tried in patients with recurrent thrombosis despite adequate anticoagulation [14-15]. Rituximab, an anti-CD20 monoclonal antibody can be used in aPL positive patients with hematological manifestations [16]. Anticoagulants, systemic glucocorticoids, plasma exchange, and intravenous immune globulin (IVIg) are generally reserved for patients with catastrophic APS [17]. Immunosuppression with cyclophosphamide or azathioprine has been reported in a very few cases of APS chorea [18-19].

Chorea in aPL is a risk marker for valvulopathy, arterial thrombotic events, and obstetric morbidity [20]. So, the early diagnosis and management of aPL-associated chorea are important to prevent these complications.

\section{Conclusions}

APS can present with a wide range of neurological manifestations. Chorea in the setting of APS is rare, and it can be an initial presentation of APS. It is important to consider APS in the differential and intervene early in the disease process to minimize the complications.

\section{Additional Information}

\section{Disclosures}

Human subjects: Consent was obtained by all participants in this study. University of Missouri IRB issued approval 2011479. The Case Report request above has been approved. Conflicts of interest: In compliance with the ICMJE uniform disclosure form, all authors declare the following: Payment/services info: All authors have declared that no financial support was received from any organization for the submitted work. Financial relationships: All authors have declared that they have no financial relationships at present or within the previous three years with any organizations that might have an interest in the submitted work. Other relationships: All authors have declared that there are no other relationships or activities that could appear to have influenced the submitted work.

\section{Acknowledgements}

We want to thank Keerthana K Kumar, MD, for suggesting edits.

\section{References}

1. Abdo WF, van de Warrenburg BP, Burn DJ, Quinn NP, Bloem BR: The clinical approach to movement disorders. Nat Rev Neurol. 2010, 6:29-37. 10.1038/nrneurol.2009.196 
2. Hughes GR: Thrombosis, abortion, cerebral disease, and the lupus anticoagulant. Br Med J (Clin Res Ed). 1983, 287:1088-1089.

3. Cervera R, Piette JC, Font J, et al.: Antiphospholipid syndrome: clinical and immunologic manifestations and patterns of disease expression in a cohort of 1,000 patients. Arthritis Rheum. 2002, 46:1019-1027. 10.1002/art.10187

4. Cervera R, Asherson RA, Font J, Tikly M, Pallarés L, Chamorro A, Ingelmo M: Chorea in the antiphospholipid syndrome. Clinical, radiologic, and immunologic characteristics of 50 patients from our clinics and the recent literature. Medicine (Baltimore). 1997, 76:203-212. 10.1097/00005792-199705000-00006

5. Abbott NJ, Mendonca LL, Dolman DE: The blood-brain barrier in systemic lupus erythematosus . Lupus. 2003, 12:908-915. 10.1191/0961203303lu5010a

6. Katzav A, Shoenfeld Y, Chapman J: The pathogenesis of neural injury in animal models of the antiphospholipid syndrome. Clin Rev Allergy Immunol. 2010, 38:196-200. 10.1007/s12016-009-8154-X

7. Soltesz P, Der H, Veres K, Laczik R, Sipka S, Szegedi G, Szodoray P: Immunological features of primary antiphospholipid syndrome in connection with endothelial dysfunction. Rheumatology (Oxford). 2008, 47:16281634. 10.1093/rheumatology/ken349

8. Asherson RA, Hughes GR: Antiphospholipid antibodies and chorea. J Rheumatol. 1988, 15:377-379.

9. Furie R, Ishikawa T, Dhawan V, Eidelberg D: Alternating hemichorea in primary antiphospholipid syndrome: evidence for contralateral striatal hypermetabolism. Neurology. 1994, 44:2197-2199. 10.1212/WNL.44.11.2197

10. Miyakis S, Lockshin MD, Atsumi T, et al.: International consensus statement on an update of the classification criteria for definite antiphospholipid syndrome (APS). J Thromb Haemost. 2006, 4:295-306. 10.1111/j.1538-7836.2006.01753.x

11. Nordal EB, Nielsen J, Marhaug G: Chorea in juvenile primary antiphospholipid syndrome. Reversible decreased circulation in the basal ganglia visualised by single photon emission computed tomography. Scand J Rheumatol. 1999, 28:324-327.

12. Orzechowski NM, Wolanskyj AP, Ahlskog JE, Kumar N, Moder KG: Antiphospholipid antibody-associated chorea. J Rheumatol. 2008, 35:2165-2170.

13. Sciascia S, Khamashta MA, D'Cruz DP: Targeted therapy in antiphospholipid syndrome . Curr Opin Rheumatol. 2014, 26:269-275. 10.1097/bor.0000000000000051

14. Petri M: Use of hydroxychloroquine to prevent thrombosis in systemic lupus erythematosus and in antiphospholipid antibody-positive patients. Curr Rheumatol Rep. 2011, 13:77-80. 10.1007/s11926-0100141-y

15. Lopez-Pedrera C, Ruiz-Limon P, Aguirre MA, Rodriguez-Ariza A, Cuadrado MJ: Potential use of statins in the treatment of antiphospholipid syndrome. Curr Rheumatol Rep. 2012, 14:87-94. 10.1007/s11926-0110222-6

16. Pons I, Espinosa G, Cervera R: Efficacy and safety of rituximab in the treatment of primary antiphospholipid syndrome: analysis of 24 cases from the bibliography review [Article in English, Spanish]. Med Clin (Barc). 2015, 144:97-104. 10.1016/j.medcli.2014.01.034

17. Cervera R, Rodriguez-Pinto I, Colafrancesco S, et al.: 14th International Congress on Antiphospholipid Antibodies Task Force Report on Catastrophic Antiphospholipid Syndrome. Autoimmun Rev. 2014, 13:699707. 10.1016/j.autrev.2014.03.002

18. Ordi-Ros J, Perez-Peman P, Monasterio J: Clinical and therapeutic aspects associated to phospholipid binding antibodies (lupus anticoagulant and anticardiolipin antibodies). Haemostasis. 1994, 24:165-174. 10.1159/000217098

19. Nordal EB, Nielsen J, Marhaug G: Chorea in juvenile primary antiphospholipid syndrome: case report. Scand J Rheumatol. 1999, 28:324-327. 10.1080/03009749950155535

20. Reiner P, Galanaud D, Leroux G, et al.: Long-term outcome of 32 patients with chorea and systemic lupus erythematosus or antiphospholipid antibodies. Mov Disord. 2011, 26:2422-2427. 10.1002/mds.23863 